\title{
The Value of Subjective Visual Vertical in Diagnosis of Vestibular Migraine* $^{*}$
}

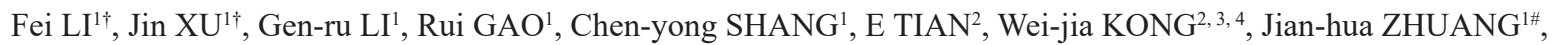 \\ Su-lin $\mathrm{ZHANG}^{2,3 \#}$ \\ ${ }^{1}$ Department of Neurology, Changzheng Hospital, Naval Medical University, Shanghai 200003, China \\ ${ }^{2}$ Department of Otorhinolaryngology, Union Hospital, Tongji Medical College, Huazhong University of Science and Technology, \\ Wuhan 430022, China \\ ${ }^{3}$ Institute of Otorhinolaryngology, Union Hospital, Tongji Medical College, Huazhong University of Science and Technology, \\ Wuhan 430022, China \\ ${ }^{4}$ Key Laboratory of Neurological Disorders of Education Ministry, Tongji Medical College, Huazhong University of Science \\ and Technology, Wuhan 430022, China
}

(C) The Author(s) 2021

\begin{abstract}
[Abstract] Objective: To study the value of the subjective visual vertical (SVV) in the diagnosis of vestibular migraine (VM). Methods: This study recruited $128 \mathrm{VM}$ patients and 64 age-matched normal subjects. We detected the SVV during the interval between attacks in both groups, in sitting upright, and the head tilted at $45^{\circ}$ to the left or right. We then examined the correlation between the SVV results with the vestibular evoked myogenic potential (VEMP) and canal paresis (CP). Results: It was found there was a significant difference in SVV at the upright position between VM patients and normal controls $(P=0.006)$ and no significant difference was found at the tilts of $45^{\circ}$ to the left or right between the two groups. The SVV results at the upright position were significantly correlated with cervical VEMP $(P=0.042)$ whereas not significantly correlated with $\mathrm{CP}$ and VEMP. There existed no significant difference in the conformity to the Müller effect (M effect) between the two groups. ROC analysis exhibited that the sensitivity, specificity of SVVs at the upright were $67.200 \%$ and $62.500 \%$ respectively. The diagnostic value of SVV at the upright position was significantly higher than that at tilts of $45^{\circ}$ to the left and right $(P=0.006)$. Nonetheless the diagnostic accuracy was relatively low. Conclusion: Abnormality in SVV possibly stems from the lasting functional disorder of cerebellar or high-level cortical centers in VM patients or is linked to the vestibular compensation. The SVV is of low diagnostic value for VM and the value of SVV in VM warrants further study.
\end{abstract}

Key words: vestibular migraine; subjective visual vertical; diagnostic value evaluation

Vestibular migraine (VM) is a common paroxysmal vestibular syndrome and, so far, the pathological mechanism of VM remain poorly understood ${ }^{[1]}$. The results of the vestibular function tests in VM patients

Fei LI, E-mail: lifei19860711@163.com; Jin XU, E-mail: candyxujin@163.com

${ }^{\dagger}$ Fei LI and Jin XU contributed equally to this work.

\#Corresponding authors, Jian-hua ZHUANG, E-mail: jianhuazh11@126.com; Su-lin ZHANG, E-mail: 156933024@qq.com

*This study was funded by grants from the Shanghai Science and Technology Commission (No. 18ZR1439200), the National Natural Science Foundation of China (No. 81873701), the Innovation Items in the Military Service Field of Changzheng Hospital (No. 2019CZJS102, No. 2020CZWJFW10) and the National Twelfth Five-Year Research Program of China (No. 2012BAI12B02). tend to vary substantially. Some VM patients presented normal results while some have findings indicating central or peripheral impairment, such as abnormal caloric test results or abnormal eye movement of central origin $^{[2-6]}$. A prior study showed that patients with migraine had sub-clinical disorders of the vestibularspinal reflex system. These abnormalities might, in part, originate from the damage to the otolith organ. These aberrant messages might affect the sensory information used for posture control, thereby resulting in postural instability ${ }^{[7]}$. Moreover, migraine-related vascular spasm might lead to inner-ear ischemia of some regions, which results in transient or permanent vestibular disorder ${ }^{[8]}$. The amplitude in cervical vestibular evoked myogenic potential (cVEMP) ${ }^{[9]}$ and ocular VEMP (oVEMP $)^{[10]}$ were lowered in VM patients and these findings support the theory that otolith 
organ impairment is implicated in VM pathogenesis. Nonetheless, some studies also found that there existed abnormalities in high-level cortical function of brain in VM patients. For instance, a study exhibited that VM patients were more sensitive to low-frequency dynamic roll tilting ${ }^{[1]}$ and an imaging study supported the assumption that the "high-level" vestibular perception was dysfunctional in VM patients ${ }^{[2]}$.

Subjective visual vertical (SVV) refers to the perceptional judgment of gravitational vertical when visual references are deprived. SVV is currently believed to be an effective technique for assessing the function of the otolith organ and the central pathways of gravity perception. It has been well established that, in normal population, when sitting upright at rest, SVV deviation should be within a range of $\pm 2.5^{\circ[13,14]}$. SVV deviation is a sensitive measure for the vestibular tone imbalance in roll plane ${ }^{[15]}$. When the vestibular periphery or nucleus sustained damage, SVV tilted to the affected side; if the damage was in or above the pons, SVV tilted contralaterally; with damage in thalamus or cerebellar dental nucleus, SVV tilted in random directions ${ }^{[16]}$. The study found that the SVV might have different presentations at different phases of condition: in patients with peripheral damage, SVV deviation might return to normal when the disease stabilizes, suggesting that central compensation might impact the SVV results ${ }^{[17]}$. At static head tilt, SVV may work better in reflecting the functions of higher-level control centers, such as the thalamus or cerebral cortex than dynamic SVV during off-vertical axis rotation ${ }^{[18]}$.

Previous studies on VM patients revealed that SVV was not altered when the head was in the upright position $^{[19,20]}$. In these patients, however, errors of upright perception have hardly been investigated during static head tilt, when the brain has to maintain a common multisensory reference frame for orientation constancy. We hypothesized that the disorder of spatial perception is associated with the disorder of gravity perception pathways of the otolith organ in VM patients. Our hypothesis is premised on the following reasoning: When the head tilts, the brain has to integrate sensory information that encodes the positions of the eye, head and body to maintain perception of upright status. As a result, SVV errors are more likely at head tilts due to the integration errors. Since the head tilt of $45^{\circ}$ is within the physiologic range of neck positions and near the upper limit of compensation $\left(60^{\circ}\right)$, if a VM patient suffers from disorder of deep and vestibular sensations, the $45^{\circ}$ head tilt suffices to bring about SVV errors.

This study examined the SVV presentations in VM patients who sat upright or tilted head $45^{\circ}$ to the right or left during the intervals of VM attacks and analyzed their association with VEMP. We aimed to understand the features of otolith impairments and provide added data for the study of the pathogenesis of VM.

\section{MATERIALS AND METHODS}

\subsection{Data and Subjects}

This study initially included 135 VM patients (meeting ICVD diagnostic criteria ${ }^{[21]}$ who visited the vertigo clinic of Changzheng Hospital of Shanghai (China) from June 2019 to December 2020. Also recruited were 68 healthy subjects with no prior history of migraine, dizziness, or other neurological disorders, serving as normal controls.

This study was approved by the hospital ethics committee of Shanghai Changzheng Hospital, Naval Medical University (CZ2019-SJNK-005) and all patients signed an informed consent for this study.

Inclusion criteria were as follows: (1) having no organic neurological or aural diseases; (2) having good neck movement; (3) having normal eyesight or corrected version of 1.0 or above; (4) electric otoscopy, pure tone audiometry, acoustic immittance measurement, video head impulse test and eye movement test yielded normal results; (5) understanding and cooperating with the relevant examinations and agreeing to sign an informed consent.

Exclusion criteria were as follows: (1) having dizziness, vertigo or hearing abnormalities not in line with the characteristics of VM; (2) having seriously aberrant vestibular functions, such as abnormalities revealed by video head impulse test and eye movement test; (3) having vertical deviation of eyes, eye diseases and serious cervical spondylopathy or limited cervical movement; (4) those who couldn't understand and cooperate during the experiment.

After elimination of ineligible candidates against the aforementioned criteria, eventually 128 patients were included. Four subjects whose SVV at upright position didn't fall into the range of reference value $\pm 2.5^{\circ}$ were excluded and, in the end, 64 healthy controls were recruited. The patient selection process is illustrated in fig. 1.

\subsection{Methodologies}

The patients and normal controls all received video nystagmography, caloric test, video head impulse test, VEMP, static SVV test. The tests were performed by senior technicians who had been engaged in vestibular examination.

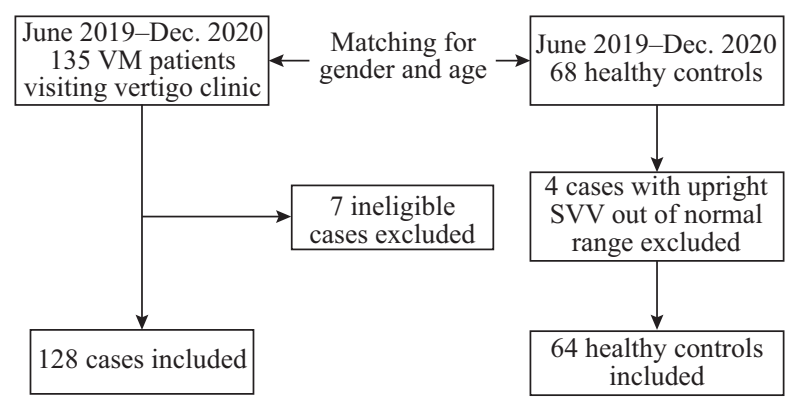

Fig. 1 Subject selection process 
Video nystagmography, caloric test, video head impulse test and VEMP were all conducted in accordance with the expert consensus on vestibular function tests (Version 2019) ${ }^{[22,23]}$.

SVV test was carried out as follows. Each subject was examined for the following fixed head positions: (a) upright head position $\left(0^{\circ}\right)$; (b) head tilt of $45^{\circ}$ to the right; (c) head tilt of $45^{\circ}$ to the left. SVV test was conducted in all three head positions. For each head position, 6 tests were performed and the average result of the 6 tests was obtained. Participants were asked to wear VR goggles (VertiGoggles ${ }^{\circledR}$ ZT-VNG-I, ZEHNIT, China). Afterwards, the participant actively avoided light and entered a dark environment. The participant could see a yellow-green light band on a black background. The light band was randomly presented to the participant at an angle in the range from $20^{\circ}$ to $90^{\circ}$, either from the right or left. The participant, by using a remote controller, turned the light band, clockwise or counterclockwise, to what he or she believed the position of "gravitational vertical" and confirmed the adjustment by pressing the "Confirm" button. In the meantime, the computer recorded his or her head position and the deviation of the light band position (in angles) from the actual gravitational vertical. For each head position, the test was repeated 6 times and the computer automatically calculated the means and the standard deviations. In the test, the computer monitored the head position in real time mode to make sure the participant kept his or her head in desired position. Under a gyroscope, the actual gravitational vertical was detected and its position defined as $0^{\circ}$. The value was recorded as positive (+) if the upper segment went to the right and the lower segment to the left and as negative (-), if the line went the opposite direction.

\subsection{Statistical Analysis}

SPSS23.0 software package was employed for statistical analysis. Measurement data were presented as mean \pm standard deviation (SD) $(\bar{x} \pm s)$ and enumeration data were expressed as percentage (\%). For measurement data, inter-group comparison was made by using homogeneity test of variance and normal distribution test. If variance was homogeneous and in normal distribution, $t$-test was used. On the other hand, if variance was not homogeneous or not in normal distribution, the rank sum test was employed. The numeration data between groups were compared by using $\chi^{2}$ test or Fisher's exact test. To set reference values, the range between 2.5 centile to 97.5 centile (95\% reference range) was taken as normal range for non-normally distributed data while for the normallydistributed data, mean \pm 1.96 standard deviation was set as the normal range $(\bar{x} \pm 1.96 \mathrm{~s})$. The correlation among analysis results was evaluated by employing Spearman rank correlation test. The receiver operating characteristic (ROC) curve was utilized for assessing the diagnostic value of SVVs at different positions in the diagnosis of VM. Test level was set at $\mathrm{a}=0.05$. A $P<0.05$ was considered to be statistically significant.

\section{RESULTS}

\subsection{Basic Features and Determination of Reference Values in the Controls}

The patient group contained 107 female subjects (83.594\%), with an average age of $52.210 \pm 11.242$ years. The control group had 47 females (73.438\%), with an average age of $48.91 \pm 10.977$ years. There were no statistically significant differences in gender $(P=0.096)$ and age $(P=0.054)$ between the two groups (table 1$)$.

Table 1 Basic features

\begin{tabular}{lrrrr}
\hline Features & Cases $(n=128)$ & Controls $(n=64)$ & $F / \chi^{2}$ & $P$ \\
\hline Age & $52.21 \pm 11.242$ & $48.91 \pm 10.977$ & 3.745 & 0.054 \\
Gender & & & & \\
Female $(n, \%)$ & $107(83.594)$ & $47(73.438)$ & 2.772 & 0.096 \\
Male $(n, \%)$ & $21(16.406)$ & $17(26.562)$ & & \\
\hline
\end{tabular}

Continuous data are shown as mean \pm standard deviation and categorical data as count (percentage). $P$-value represents the comparison between cases and controls.

Our tests showed that, in the healthy controls, the values at tilt of $45^{\circ}$ to the right and values at tilt of $45^{\circ}$ to the left all conformed to a normal distribution $(P>0.05)$. The $95 \%$ reference range of the right title at $45^{\circ}$ was $(-15.125,7.807)$ and the $95 \%$ reference range of the left tilt at $45^{\circ}$ was $(-10.048,15.174)$ (table 2 ). Against the $95 \%$ reference ranges of values at the upright position, tilt of $45^{\circ}$ to the right and head tilt of $45^{\circ}$ to the left right, we found that the upright head position had a higher error rate (47 cases, accounting for $36.719 \%$ ). Tilt of $45^{\circ}$ to the right and tilt of $45^{\circ}$ to the left yielded significantly lower error rates $(n=21$, $16.406 \%$ with the right tilt and, $n=16,12.500 \%$ with the left tilt, respectively).

Table 2 Normality test and $95 \%$ reference range in healthy controls

\begin{tabular}{|c|c|c|c|c|c|c|c|c|}
\hline \multirow{2}{*}{ Positions } & \multicolumn{3}{|c|}{ Normality test } & \multicolumn{3}{|c|}{ Quartile test } & \multirow{2}{*}{ Mean } & \multirow{2}{*}{ SD } \\
\hline & Skewness & Kurtosis & $P$ & Median & $P_{2.5}$ & $P_{97.5}$ & & \\
\hline Upright position & -0.395 & -0.917 & 0.002 & 0.450 & -2.313 & 2.275 & 0.223 & 1.260 \\
\hline Tilt of $45^{\circ}$ to right & -0.224 & 0.144 & 0.200 & -3.800 & -17.800 & 7.938 & -3.659 & 5.850 \\
\hline Tilt of $45^{\circ}$ to left & 0.020 & -0.155 & 0.200 & 2.200 & -10.700 & 15.688 & 2.563 & 6.434 \\
\hline
\end{tabular}

To set the reference values at SVV of the normal control group, the normal distribution test and the quartile test of the data were carried out. Normality test: normal distribution test. $P_{2.5}$ : data of 2.5 centile. $P_{97.5}:$ data of 97.5 centile. SD: standard deviation 
Analysis revealed that there existed no correlation in SSV test results among the three head positions, i.e., the upright position, the tilts of $45^{\circ}$ to the right and the left. Moreover, no correlation was found between the SVV results and the age (table 3).

\subsection{Comparison between Patients and Controls in Terms of SSV Results at Three Head Positions}

There were significant differences in the SVV

Table 3 Correlation between SVV test results and three head positions in the controls

\begin{tabular}{llrr}
\hline Indicator 1 & Indicator 2 & $\begin{array}{c}\text { Spearman } \\
\text { correlation } \\
\text { coefficient }\end{array}$ & $P$ \\
\hline Age & Upright position & -0.11 & 0.386 \\
& Tilt of $45^{\circ}$ to the right & 0.227 & 0.072 \\
& Tilt of $45^{\circ}$ to the left & -0.218 & 0.083 \\
Upright position & Tilt of $45^{\circ}$ to the right & 0.123 & 0.331 \\
& Tilt of $45^{\circ}$ to the left & 0.243 & 0.053 \\
Tilt of $45^{\circ}$ to the right & Tilt of $45^{\circ}$ to the left & -0.168 & 0.185 \\
\hline
\end{tabular}

results at the upright position between the two groups $(P=0.006)$, with the values in the patients being significantly less than those in the controls. There were also significant differences in the absolute values of the three positions between the two groups $(P=0.003)$. No significant difference was revealed between the two groups when the head tilted $45^{\circ}$ to the left or right (table 4).

Analysis showed that there were no significant differences in the conformity to the Müller (M effect) between the two groups, neither unilaterally nor bilaterally $(P>0.05)$ (table 5).

\subsection{Correlation between SVV Test Results and CP, cVEMP, oVEMP in Patients}

Our analysis exhibited that only SVV results at the upright position were significantly correlated with cVEMP $(P=0.042)$. SVVs at tilts of $45^{\circ}$ to the right and the left were not significantly correlated with $C P$, cVEMP and oVEMP (table 6).

Table 4 Comparison between patients and controls in terms of test result errors

\begin{tabular}{llccc}
\hline Positions & Groups & Median (lower and upper quartiles) & $Z$ & $P$ \\
\hline \multirow{2}{*}{ Upright } & Patients & $-0.700(-2.500,1.175)$ & -2.769 & 0.006 \\
& Controls & $0.450(-0.800,1.300)$ & & \\
$45^{\circ}$ tilt to right & Patients & $-5.00(-9.600,0.100)$ & -1.404 & 0.160 \\
& Controls & $-3.800(-6.800,0.300)$ & & \\
$45^{\circ}$ tilt to left & Patients & $2.100(-2.500,7.425)$ & -0.264 & 0.791 \\
\multirow{2}{*}{ Absolute values at three positions } & Controls & $2.200(-1.100,7.250)$ & & \\
& Patients & $15.100(9.025,20.825)$ & -2.995 & 0.003 \\
\hline
\end{tabular}

Table 5 Conformity to the $M$ effect in two groups

\begin{tabular}{lcccc}
\hline Conformity & $\begin{array}{c}\text { Patients } \\
(n, \%)\end{array}$ & $\begin{array}{c}\text { Controls } \\
(n, \%)\end{array}$ & $\chi^{2}$ & $P$ \\
\hline Yes at right side & $95(74.219)$ & $47(73.437)$ & 0.014 & 0.907 \\
Yes at left side & $83(64.844)$ & $47(73.437)$ & 0.574 & 0.449 \\
Yes at both sides & $70(54.687)$ & $36(56.250)$ & 0.042 & 0.837 \\
Not at neither side & $20(15.625)$ & $8(12.500)$ & 0.334 & 0.563 \\
\hline$P$-value represents the comparison on the conformity of the
\end{tabular}

Table 6 Correlation between SVV and the peripheral vestibular function in patients

\begin{tabular}{llcc}
\hline Indicator 1 & Indicator 2 & $\begin{array}{c}\text { Spearman } \\
\text { correlation } \\
\text { coefficient }\end{array}$ & $P$ \\
\hline \multirow{3}{*}{ Upright position } & CP & 0.026 & 0.791 \\
& cVEMP & 0.199 & 0.042 \\
& oVEMP & -0.033 & 0.745 \\
Tilt of $45^{\circ}$ to the right & cP & 0.055 & 0.579 \\
& oVEMP & -0.043 & 0.660 \\
Tilt of $45^{\circ}$ to the left & CPEMP & -0.040 & 0.691 \\
& oVEMP & -0.016 & 0.875 \\
& & 0.081 & 0.615 \\
\hline
\end{tabular}

$\overline{\mathrm{CP}}$ : canal paresis; cVEMP: cervical vestibular evoked myogenic potential; oVEMP: ocular vestibular-evoked myogenic potential

\subsection{Value of SVV Test in the Diagnosis of VM}

Our analysis showed that the sensitivity and specificity of SVV test at upright position for the diagnosis of VM were $67.200 \%$ and $62.500 \%$, respectively. The area under the ROC curve (AUC) was $0.623(0.545-0.700)$ and cut-off value was -0.350 . The diagnostic value of SVV was significantly higher at the upright position than at tilts of $45^{\circ}$ to the right or left $(P=0.006)$. Since $\mathrm{AUC}<0.7$, the diagnostic accuracy was relatively low (table 7 ). The working curve analysis revealed that even adding the absolute values at the three positions together failed to increase the diagnostic power $(\mathrm{AUC}<0.7)$ (table 7) (fig. 2).

\section{DISCUSSION}

Patients with VM often complain of dizziness with changes in the head or body position. Such symptoms mean the spatial orientation may not function properly in these patients. Currently, it is generally believed that any abnormality along the otolith pathways, including utricle, saccule, brain stem, thalamus, vestibular cortext, leads to errors of spatial orientation ${ }^{[2]}$. Mechanistically, the development and progression of $\mathrm{VM}$ involve both peripheral vestibular system and central nerve 
Table 7 The value of SVV test for the VM diagnosis

\begin{tabular}{lcccccc}
\hline Indicators & AUC & Sensitivity & Specificity & cut-off value & $P$ & $95 \%$ IC \\
\hline Upright & 0.623 & 0.672 & 0.625 & -0.350 & 0.006 & $0.545-0.700$ \\
Tilt of $45^{\circ}$ to right & 0.562 & 0.766 & 0.422 & -6.900 & 0.160 & $0.480-0.644$ \\
Tilt of $45^{\circ}$ to left & 0.512 & 0.766 & 0.322 & -1.250 & 0.791 & $0.427-0.597$ \\
Sum of the absolute of values at three positions & 0.633 & 0.484 & 0.797 & 15.650 & 0.003 & $0.554-0.712$ \\
\hline
\end{tabular}

AUC: area under ROC curve. 95\% IC: 95\% confidence interval

A

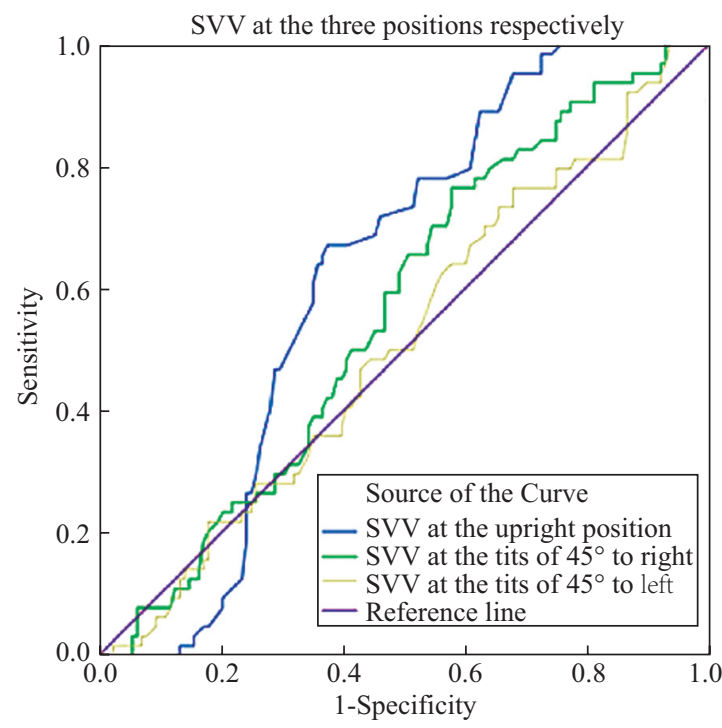

B

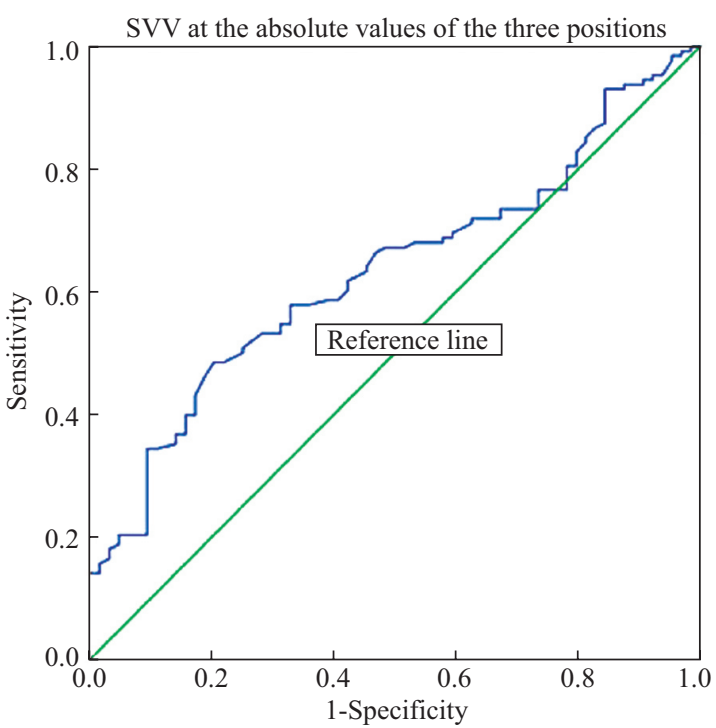

Fig. 2 ROC curves of SVV in the study

A: SVV at the three positions respectively. blue line, SVV at the upright position; green line, SVV at the tilts of $45^{\circ}$ to right; pink line, SVV at the tilts of $45^{\circ}$ to left; purple line, reference line. B: SVV at the absolute values of the three positions, blue line. The green line is reference line.

system $^{[25]}$. To further understand the features of otolith pathway damage in VM patients, this study examined the static SVV at the upright position, at head tilts of $45^{\circ}$ to the right and left during intervals of $\mathrm{VM}$ attacks and compared the SVV results with findings of VEMP and CP.

According to previous reports, in normal populations, the deviation of the static SVV at the upright position should be within $\pm 2.5^{\circ[13,18]}$. Nonetheless, no consensus has been arrived at concerning the normal range of SVV at tilts. In this study, we recruited subjects, as normal controls, who previously had no dizziness, vertigo, migraine and other neurological, otological and ophthalmological conditions and whose SVV at the upright position was within $\pm 2.5^{\circ}$. Our detection showed that the $95 \%$ reference range of the right tilt at $45^{\circ}$ was $(-15.125$, $7.807)$ and the $95 \%$ reference range of the left tilt at $45^{\circ}$ was $(-10.048,15.174)$ (table 2$)$. The error rates at the upright position and at tilts of $45^{\circ}$ to the right and the left were $36.719 \%, 16.406 \%, 12.500 \%$, respectively. There was statistically significant difference in the SVV result at the upright position between the two groups $(P=0.006)$ and the SVV values were significantly less in patients than in controls, i.e., the perception of VM patients to the gravitational vertical tended to tilt to the left at the upright position. No statistically significant difference was found in the SVV result at tilts of $45^{\circ}$ to the right or left between the two groups. In this study, VM patients had higher abnormality rate in terms of SVV at the upright position, with diagnostic sensitivity and specificity being $67.200 \%$ and $62.500 \%$, respectively, suggesting that it can serve as a complementary indicator for the diagnosis of VM. The conclusion reached in this study is not fully consistent with the conclusions of previous researches on SVV tests in VM patients. Prior studies found no SVV deviation in VM patients at the upright position ${ }^{[19}$, ${ }^{20]}$. This discrepancy might be attributed to the phase of the condition during which the patients were recruited. In our cohort, most patients had clinical VM episodes 1 week before the recruitment and some even had attacks 1 day before SVV test. We retrieved only one study which found that in patients whose symptoms persisted for over 24 hours ${ }^{[26]}$. Given the differences between the two studies in patient selection and symptoms, which indicated that the mechanisms and locations involved in the two studies were different, we believe that this discrepancy might also be ascribed to the sample selection bias. Moreover, this study showed that there was no significant difference in SVV at tilt of $45^{\circ}$ between VM patients and controls. On the other hand, 
Winnick et al found that the static SVV deviation at a tilt of $20^{\circ}$ was less in VM patients than in normal subjects $^{[27]}$. Our finding was not in agreement with their results. The study by Winnick et al included only $27 \mathrm{VM}$ patients and their sample size was small and sampling errors were very alike. In addition, their tilt angle $\left(20^{\circ}\right)$ differed from ours $\left(45^{\circ}\right)$. The two factors might largely explain the discrepancy between the two studies.

To further know if SVV abnormalities result from vestibular damage of peripheral or of central origin, we examined the correlations among CP, oVEM, cVEMP and the SVVs at three head positions. Our study demonstrated that SVV at the upright position was correlated with cVEMP $(P=0.042)$. Considering that cVEMP is indicative of function of saccular pathways whereas SVV reflects, to a greater extent, the function utricular pathways, we believe its clinical implications await further exploration. Moreover, in this study, CP, which measures the function of semicircular canal, and oVEMP, an indicator of utricular function, bore no significant correlation with SVVs at the three positions, suggesting that SVV abnormalities in VM patients are not directly linked to the functions of the semi-circular canal and utricule, indicating that SVV abnormalities could not be solely attributed to the damage of peripheral otolith organs. VM patients had no signs of vestibular or ocular motor dysfunction that could lead to abnormal SVV deviations. This prompted us to hypothesize that SVV deviations in these patients might be related to a "higher-order" dysfunction in multisensory integration for spatial orientation and to the vestibular compensation secondary to peripheral damage. This hypothesis is mechanistically in line with the potential role that the multisensory integration plays in migraine pathophysiology ${ }^{[28]}$.

In a dark room, a normal subject develops delusion when he or she inclines head about the nasal-occipital axis, towards to the left or right shoulder and when his or her body system tries to adjust subjective vertical to the gravitational vertical. When the head tilt is less than $60^{\circ}$, the subjective vertical is away from the head tilt direction while the tilt angle is greater than $60^{\circ}$, the subjective vertical is in line with the head tilt direction. Howard dubbed these two phenomena as Müller (M) effect and Aubert (A) effect ${ }^{[29,30]}$.

In this study, SVV test was conducted in static state, with a head tilt of $45^{\circ}$ and comparison was made between two groups to see if they were in conformity with $\mathrm{M}$ effect. Our results revealed no significant differences in the conformity with the $\mathrm{M}$ effect between the two groups, neither unilaterally nor bilaterally. These results suggested that during the interval of VM episodes, adding input of deep sensation information did not increase the error rate of verticality perception. Given that there were no significant differences in
SVV values at head tilts between patients and healthy controls, we believe that the deep sensation does not weigh heavily in the disorder of spatial orientation in VM patients. The errors in sensory integration of the spatial orientation stem more from the disorder of signal redundancy processing of visual or vestibular senses.

This study also found that there was a substantial individual variation in SVV results among VM patients. We inferred that, if the patients in our series had been further sub-grouped in terms of clinical features, in terms of type of dizziness, presence or absence of visual vertigo, tolerance or intolerance to head movement, presence or lack of premonitory symptoms, the subgroup analysis might have yielded more valuable findings. Nonetheless, the sample size of our cohort was too small to allow further sub-grouping. This is a limitation of the study.

To sum up, this study found that SVV exerted complementary value for the diagnosis of VM. Nevertheless, since the ROC analysis showed that the AUC was less than 0.7, its diagnostic accuracy is low. So far, we don't recommend that SVV result can be used as an indicator for the diagnosis of VM and further studies are warranted to further explore the value of SVV in the diagnosis of VM.

\section{Open Access}

This article is licensed under a Creative Commons Attribution 4.0 International License https://creativecommons.org/licenses/by/4.0/), which permits use, sharing, adaptation, distribution and reproduction in any medium or format, as long as you give appropriate credit to the original author(s) and the source, provide a link to the Creative Commons licence, and indicate if changes were made. The images or other third party material in this article are included in the article's Creative Commons licence, unless indicated otherwise in a credit line to the material. If material is not included in the article's Creative Commons licence and your intended use is not permitted by statutory regulation or exceeds the permitted use, you will need to obtain permission directly from the copyright holder. To view a copy of this licence, visit http://creativecommons. org/licenses/by/4.0/.

\section{Conflict of Interest Statement}

All authors declare no conflicts of interest.

Author Su-ling ZHANG is a young member of the Editorial Board for Current Medical Science. The paper was handled by the other Editor and has undergone rigrous peer review process. Author Su-ling ZHANG was not involved in the journal's review of, or decisions related to, this manuscript.

\section{REFERENCES}

1 Juan ME, Jose AL. New insights into pathophysiology of vestibular migraine. Front Neurol, 2015,6(6):12

2 Marianne D, Mark O, Nese C. Vestibular migraine: the most frequent entity of episodic vertigo. J Neurol, 2016, 
263(S1):S82-89

3 Sohn JH. Recent Advances in the Understanding of Vestibular Migraine. Behav Neurol, 2016,2016:1801845

4 Jung ES, Kim CH, Hong JP. Vestibular abnormality in patients with Meniere's disease and migrainous vertigo. Acta Otolaryngol, 2013,133(2):154-158

5 Michael VB, Daniel Z, Hannelore N, et al. Acute migrainous vertigo: clinical and oculographic findings. Brain, 2005,128(Pt 2):365-374

6 Sharon HP, Ronald JT. Nystagmus during attacks of vestibular migraine: an aid in diagnosis. Audiol Neurootol, 2010,15(4):241-246

7 Thomas B, Michael S. General vestibular testing. Clin Neurophysiol, 2005,116(2):406-426

8 Lee H, Lopez I, Ishiyama A, et al. Can migraine damage the inner ear? Arch Neurol, 2000,57(11):1631-1634

9 Bernhard B, Stieber N, Dieterich M. Vestibular-evoked myogenic potentials in vestibular migraine. J Neurol, 2009,256(9):1447-1454

10 Zuniga MG, Kristen LJ, Michael CS, et al. Can vestibular-evoked myogenic potentials help differentiate Ménière disease from vestibular migraine? Otolaryngol Head Neck Surg, 2012,146(5):788-796

11 Joseph MF, Dawn AM. Migraine and motion sensitivity. Continuum (Minneap Minn), 2012,18(5 Neuro-otology): 1102-1117

12 Jung HS, Yu KK, Hyo JK, et al. Altered brain metabolism in vestibular migraine: Comparison of interictal and ictal findings. Cephalalgia, 2014,34(1):58-67

13 Christopher JD, Amy P, Paola G, et al. Cerebellar Degeneration Increases Visual Influence on Dynamic Estimates of Verticality. Curr Biol, 2018,28(22):35893598

14 Faith WA, Owen DM, Amber P, et al. Normative data for the subjective visual vertical test during centrifugation. J Am Acad Audiol, 2011,22(7):460-468

15 Eghlimi B, Schaaf H, Hesse G, et al. Measuring the subjective visual vertical using a portable system: a comparison with the standard darkroom method. HNO, 2012,60(4):330-336

16 Maristela MF, Maurício MG, Heloisa HC. Subjective visual vertical after treatment of benign paroxysmal positional vertigo. Braz J Otorhinolaryngol, 2017,83(6): 659-664

17 Clarke AH, Schönfeld U, Hamann C, et al. Measuring unilateral otolith function via the otolith-ocular response and the subjective visual vertical. Acta Otolaryngol
Suppl, 2001,545:84-87

18 Faith WA, Owen DM, Amber P, et al. Normative data for the subjective visual vertical test during centrifugation. J Am Acad Audiol, 2011,22(7):460-468

19 Masayuki A, Mitsuhiro A, Hisamitsu H, et al. Subclinical deviation of the subjective visual vertical in patients affected by a primary headache. Acta Otolaryngol, 2009,129(1):30-35

20 Luc C, Leen V, Anouk V. Patients with migraine correctly estimate the visual verticality. Clin Neurol Neurosurg, 2012,114(4):313-315

21 Thomas L, Jes O, Joseph F, et al. Vestibular migraine: diagnostic criteria. J Vestib Res, 2012,22(4):167-172

22 Toshihisa M, Masahito T, Kyoko K, et al. Simultaneous Presentation of Definite Vestibular Migraine and Definite Ménière's Disease: Overlapping Syndrome of Two Diseases. Front Neurol, 2018,10(9):749

23 Toshihisa M, Haruka N, Eriko Y, et al. Association of air-conducted sound oVEMP findings with cVEMP and caloric test findings in patients with unilateral peripheral vestibular disorders. Acta Otolaryngol, 2011,131(9): 945-950

24 Friedmann G. The judgment of the visual vertical and horizontal with peripheral and central vestibular lesions. Brain, 1970,93(2):313-328

25 Koo JW, Balaban CD. Serotonin-induced plasma extravasation in the murine inner ear: possible mechanism of migraine-associated inner ear dysfunction. Cephalalgia, 2006,26(11):1310-1319

26 Chang TP, Ariel AW, Yung $\mathrm{CH}$, et al. The bucket test differentiates patients with MRI confirmed brainstem/ cerebellar lesions from patients having migraine and dizziness alone. BMC Neurol, 2019,19(1):219

27 Ariel W, Shirin S, Jorge OM, et al. Errors of Upright Perception in Patients With Vestibular Migraine. Front Neurol, 2018,30(9):892

28 Todd JS. Multisensory integration in migraine. Curr Opin Neurol, 2013,26(3):248-253

29 Daniel B, Christopher JB, Dominik S, et al. Differential effects of visual feedback on subjective visual vertical accuracy and precision. PLoS One, 2012,7(11):e49311

30 Maristela MF, Maurício MG, Heloisa HC. Subjective visual vertical after treatment of benign paroxysmal positional vertigo. Braz J Otorhinolaryngol, 2017,83(6): 659-664

(Received May 31, 2021; accepted Jul. 30, 2021) 